Revista Eletrônica do Mestrado

Profissional em Administração da UnP

\title{
edunp
}

V. 11, N. 2, 2019

ISSN 1984-4204

https://repositorio.unp.br/index.php/raunp

https://doi.org/10.21714/raunp.v11i2.2074

\section{Propostas metodológicas de ensino: qual a importância de propostas inovadoras ou tradicionais?}

\section{Methodological proposals of teaching: how important are innovative or traditional proposals?}

\author{
Daiane do Amaral ${ }^{\mathrm{a}}$, Rosana da Rosa Portella Tondolo ${ }^{\mathrm{b}}$, Vilmar Antonio Gonçalves Tondolo ${ }^{\mathrm{c}}$, Maira Bernardi ${ }^{\mathrm{d}}$ \\ ${ }^{a}$ Mestra em Administração Pública. Universidade Federal de Pelotas (UFPEL), Brasil. daianeamaral26@gmail.com \\ b Professora da Universidade Federal de Pelotas (UFPEL), Brasil. rosanatondolo@gmail.com \\ c Professor da Universidade Federal de Pelotas (UFPEL), Brasil. vtondolo.ufpel@gmail.com \\ ${ }^{d}$ Doutora em Educação/UFRGS. Núcleo de Tecnologia Digital aplicada à Educação (NUTED/UFRGS). mairaber@gmail.com
}

\begin{abstract}
Resumo
O presente estudo teve como objetivo identificar como as metodologias de ensino adotadas influenciam a intenção dos acadêmicos de uma Instituição de Ensino Superior da região sul do Brasil em cursar um curso superior de tecnologia na área de gestão. Para atender ao objetivo, foi empregado um experimento baseado em cenários com a utilização de vinhetas. Após a apresentação das vinhetas, os acadêmicos responderam a um questionário sobre a intenção de realizar o curso apresentado. A amostra foi composta por 85 acadêmicos de um curso superior de tecnologia do sul do Brasil. Os dados da pesquisa foram analisados de forma quantitativa por meio da técnica estatística de análise de variância. Como resultados, verificou-se que a adoção de diferentes metodologias de ensino possui influência sobre a intenção dos alunos em realizar o curso apresentado, o que demonstra a importância da análise dessa influência, diante das profundas transformações pelas quais vem passando o processo educacional ao longo do tempo.
\end{abstract}

Palavras-chave: Metodologias de Ensino; Intenção de cursar; Ensino Superior

\begin{abstract}
The present study had as objective to identify how the teaching methodologies adopted influence the intention of the academics of a Higher Education Institution of the southern region of Brazil to attend a higher technology course in the area of management. In order to achieve the main goal, a scenario-based role-playing experiment using vignettes was employed. After the presentation of the vignettes, the academics answered a questionnaire about the intention to take the course presented. The sample consisted of 85 academics from a technology course in southern Brazil. The data of the research were quantitatively analyzed through the statistical technique of analysis of variance. As results, it was verified that the adoption of different teaching methodologies has influence on the intention of the students to carry out the course presented, which demonstrates the importance of the analysis of this influence, given the profound transformations that the educational process has been going through of time.
\end{abstract}

Keywords: Teaching Methodologies; Intention to enroll; Higher education 


\section{Introdução}

As últimas décadas foram caraterizadas por um aumento na oferta e na flexibilidade de modalidades de ensino presentes na formação, tanto na graduação quanto na pós-graduação, o que acarretou um aumento significativo no número de alunos no ensino superior (Araújo, Santos, Noronha, Zanon, Ferreira, Casanova, \& Almeida, 2016). Isso foi importante para que mais alunos pudessem ter acesso à educação de nível superior, mas também possibilitou o surgimento de novas discussões. Assim, questões sobre a transição e adaptação acadêmica dos estudantes que ingressam no ensino superior ganharam relevância nos últimos anos, uma vez que a democratização do acesso deve ser acompanhada de medidas favoráveis ao sucesso dos estudantes por parte das instituições (Almeida, Araújo, \& Martins, 2016).

Além disso, com as mudanças ocorridas na sociedade e a crescente falta de estímulo dos estudantes em participar das aulas, torna-se fundamental o desenvolvimento de novas práticas educacionais (Teles, Silva, \& Maciel, 2012). O perfil dos alunos está mudando, por isso, é importante a inclusão de atividades que despertem seu interesse. Nesse contexto, a inserção de novas metodologias é vista como uma possibilidade de ampliar e diversificar as formas de construir o conhecimento dos alunos (Teles et al., 2012).

As dificuldades de aprendizado e as formas de adaptação do sistema de ensino também são temas relevantes, uma vez que o ensino só apresenta um sentido quando implica na aprendizagem, sendo necessário conhecer como o professor ensina, além de entender como o aluno aprende (Tabile \& Jacometo, 2017). Assim, tendo em vista que a aprendizagem é influenciada por diversos fatores, é importante conhecê-los melhor para entender como o processo pode ser desenvolvido de forma mais eficaz.

Os resultados da aprendizagem, em relação às metodologias de ensino-aprendizagem e aos processos de avaliação adotados, também tem sido alvo de estudos (Biggs, 1999; Vigaray, Lopez, Peris, Martínez, Cuevas, Posadas, \& Vallés, 2010), o que demonstra a importância deste tema diante das constantes mudanças pelas quais a sociedade vem passando ao longo dos anos. Além disso, conforme Alves, Rolon, Petry, Farias, Dick, \& Melo (2018), que avaliaram as publicações sobre a temática de metodologias ativas entre os anos 2009 e 2017, com foco nos cursos de administração, tais estudos na área de gestão apesar de virem aumentando ao longo dos anos, ainda estão em seu estágio inicial, abrindo, assim, uma agenda para pesquisa.

E é nesse contexto que o presente estudo se desenvolve, apresentando um recorte da pesquisa de mestrado de mesmo nome, a qual objetivou identificar como as metodologias de ensino adotadas influenciam a intenção dos acadêmicos de uma Instituição de Ensino Superior (IES) da região sul do Brasil em cursar um curso superior de tecnologia na área de gestão. Para isso, foi realizado um experimento baseado em cenários, por meio de vinhetas, para simular a criação de um novo curso, o qual foi apresentado aos respondentes em dois formatos, utilizando metodologias de ensino distintas.

O estudo justifica-se pela importância de se conhecer as metodologias de ensino consideradas mais atraentes pelos alunos, influenciando em sua intenção de escolha, uma vez que eles são o público alvo das instituições de ensino. Além disso, "o processo de ensino-aprendizagem é um tema muito complexo e abre caminhos para a realização de várias pesquisas" (Morozini, Cambruzzi, \& Longo, 2007, p. 100). Aliado a isso, verifica-se que as atitudes interferem na intenção comportamental dos futuros acadêmicos no processo de escolha do serviço educacional (Sousa, Neto, \& Fontenele, 2013), o que também é ressaltado por Souza (2009) quando sugere a busca pela compreensão das dimensões de atitudes frente à escolha por ensino superior. 


\section{Fundamentação teórica}

\subsection{Cursos superiores de tecnologia}

A educação superior tem passado por importantes transformações e remodelações ao longo dos anos, muitas das quais envolvendo a educação tecnológica (Favretto \& Moretto, 2013). Nesse contexto, os Cursos Superiores de Tecnologia, os quais representam uma parcela significativa do total de cursos de graduação, apresentaram um expressivo crescimento na oferta na última década (Favretto \& Moretto, 2013).

De acordo com o Catálogo Nacional dos Cursos Superiores de Tecnologia, existem 134 cursos dessa modalidade sendo oferecidos no Brasil, os quais estão estruturados em 13 eixos tecnológicos (MEC, 2016). Conforme o referido catálogo, nas edições de 2006 e 2010 foram elencados 98 e 113 cursos, respectivamente, o que demonstra o efetivo crescimento de cursos nessa modalidade no país nos últimos anos (MEC, 2016). Além disso, os cursos de educação profissional tecnológica de graduação não vêm somente crescendo e se solidificando nos últimos tempos, mas também ganhando reconhecimento e aceitação gradativa no mercado de trabalho e na sociedade (Takahashi, 2010).

Assim, a educação profissional e tecnológica surge como um esforço estratégico frente às constantes mudanças socioeconômicas e políticas do país, uma vez que a metodologia adotada nessa modalidade de ensino possui foco na aprendizagem, no saber e no saber-fazer, além de adotar propostas didático-pedagógicas voltadas para a prática (Takahashi, 2010).

Nesse contexto de expansão, os cursos de tecnologia necessitam cada vez mais de uma articulação entre conhecimento científico, trabalho e educação, por isso, se faz necessária uma abordagem pedagógica orientada para que o sujeito possa ser o protagonista na construção ativa de seu conhecimento e nas relações e interações com os outros e com o mundo (Mello, 2007). Além disso, uma diferença significativa entre o bacharelado e o tecnólogo é que o último se enquadra num perfil mais conciso com relação à carga horária, além de ter um direcionamento mais prático e ser mais focado em certa área (Mello, 2007).

\subsection{Metodologias de ensino e processo educacional}

$\mathrm{Na}$ organização do processo de ensino, a didática, enquanto "área da pedagogia que estuda a técnica de ensino em todos os seus aspectos, desde a organização até a prática", tem um papel importante, sendo complementada pela metodologia de ensino no que se refere aos meios utilizados para apresentar o conteúdo e avaliar os alunos (Teixeira, 2015, p. 21). Essa estruturação do processo educacional, então, é decorrente de um planejamento que visa a escolha dos conteúdos, procedimentos e estratégias que deverão ser adotados para um melhor aproveitamento dos alunos (Ferraz \& Belhot, 2010). A metodologia, assim, abrange os métodos, as técnicas e as estratégias de ensino que o professor utiliza em sua prática docente, visando facilitar o processo de aprendizagem (Teixeira, 2015).

As IES devem buscar constantemente o aprimoramento na prestação dos serviços educacionais e nas formas de transmissão do conhecimento aos acadêmicos. Para isso, a organização curricular dos cursos deve ser permanentemente atualizada pelas instituições e deve primar pelo uso de metodologias que integrem a vivência e a prática profissional ao longo do processo formativo (Mello, 2007). Além disso, devem ser adotadas estratégias de ensino capazes de atrair a atenção dos alunos para o conteúdo a ser estudado, além da proposição de atividades que apresentem desafios e despertem a curiosidade dos estudantes (Silva, 2014), uma vez que o grande desafio da atualidade é a busca por estratégias que ajudem a reverter a falta de motivação por 
parte dos alunos, além de identificar suas razões, buscando assim uma maior qualidade no processo de ensinoaprendizagem (Tabile \& Jacometo, 2017).

Tendo em vista a importância das metodologias de ensino nesse processo, a análise de sua adoção torna-se fundamental para um melhor entendimento do processo educacional, uma vez que, conforme Andere (2007), os problemas com a qualidade do ensino podem estar relacionados à eficácia das metodologias de ensino utilizadas pelas instituições. Nesse contexto, o ensino superior pode ser visto como uma teia complexa de aptidões, conhecimentos e competências, onde as preparações, por melhores que sejam, necessitam de atualização, uma vez que os estudantes e suas atitudes com relação à aprendizagem mudam com o passar do tempo (Ramos, Delgado, Afonso, Cruchinho, Pereira, Sapeta, \& Ramos, 2013).

Por isso, conforme já observado por Morozini et al. (2007), permanece sendo necessário que o ensino evolua, visando a melhoria do processo ensino-aprendizagem. Mas, para isso, torna-se indispensável o conhecimento dos fatores que influenciam o desempenho dos alunos em sala de aula, uma vez que somente desta forma será possível obter uma proposta de alternativas que possam contribuir para a melhoria do processo tendo por base a realidade vivenciada diariamente pelos acadêmicos (Morozini et al., 2007).

Mas, para a escolha dos métodos a serem utilizados em cada caso, é importante verificar as habilidades que os alunos devem adquirir, além de se ter em mente a disciplina à qual o módulo pertence e o contexto organizacional da instituição onde ele é ministrado (Vigaray et al., 2010). Além disso, para se alcançar uma mudança de paradigma no processo de ensino-aprendizagem, além da escolha do método mais adequado, é importante especificar as tarefas que os alunos devem realizar, pois isso possibilita que eles se tornem os protagonistas de seu próprio processo de aprendizagem (Vigaray et al., 2010). Assim, é importante ressaltar que não há um método melhor ou pior, pois deve-se analisar cada situação para identificar quais métodos podem ser usados, e quais não podem, além de se verificar a possibilidade de uma combinação entre diferentes métodos (Vigaray et al., 2010).

\subsection{Metodologias tradicionais e inovadoras}

É cada vez mais difícil conseguir relacionar as práticas pedagógicas e profissionais quando se utiliza o sistema tradicional de ensino, já que esse modelo é caracterizado pela utilização de métodos expositivos, os quais baseiam-se essencialmente na ação do docente (Ramos et al., 2013). Mas, mesmo sendo alvo de críticas, as metodologias de ensino tradicionais apresentam resultados, pois, caso contrário, as aulas teóricas não seriam mais utilizadas na atualidade por parte dos professores (Krüger \& Ensslin, 2013). O problema, porém, é que sozinhas essas metodologias já não conseguem dar conta de repassar todo o conhecimento sem que o estudo se torne, por vezes, maçante para os alunos. Nesse contexto, o ensino centrado no estudante pode ser aplicado como complemento ao método tradicional, fazendo com que o aluno se torne mais ativo na busca pelo conhecimento (Krüger \& Ensslin, 2013).

Porém, na maioria das vezes os cursos de ensino superior possuem um formato engessado, onde os alunos acabam ficando restritos ao que o sistema impõe, mesmo possuindo autonomia e maturidade (Brandão, Cavalcante, \& Temoteo, 2014). Além disso, os métodos de ensino são focados em processos de memorização e não relacionam a teoria com a prática, focando apenas na transferência de conteúdo, não sendo suficientes para garantir o ensino e a aprendizagem de forma eficiente (Marques, Message, Gitahy, \& Souza, 2018). No Quadro 1, é apresentada uma síntese das metodologias tradicionais. 


\begin{tabular}{|l|l|}
\hline \multicolumn{1}{|c|}{ Metodologia } & \multicolumn{1}{c|}{ Síntese } \\
\hline Aulas Expositivas & $\begin{array}{l}\text { Visa a introdução de novos conceitos, a transmissão de informações e a exposição de } \\
\text { conhecimentos para os alunos. }\end{array}$ \\
\hline Resolução de exercícios & Busca a resolução de um problema fictício a partir do conteúdo apresentado. \\
\hline Utilização de apostilas & Visa a apresentação dos conteúdos das disciplinas. \\
\hline Proposição de exercícios & $\begin{array}{l}\text { Busca identificar se os alunos conseguem resolver o problema proposto tendo por base o } \\
\text { conteúdo da aula. }\end{array}$ \\
\hline
\end{tabular}

Quadro 1. Síntese Metodologias de Ensino Tradicionais

Fonte: Adaptado

Assim, se faz necessária a adoção de metodologias que privilegiem o protagonismo do aluno na construção do conhecimento (Silva, 2017), uma vez que o contexto dinâmico no qual a educação está inserida exerce forte pressão para que as instituições de ensino busquem uma transformação e adaptação às atuais necessidades (Signori, Guimarães, Severo, \& Rotta, 2018). Além disso, os recentes avanços tecnológicos proporcionaram uma série de inovações no campo acadêmico e educacional e trouxeram mudanças significativas nos processos globais de aprendizagem (Signori et al., 2018). Nesse contexto, métodos práticos de ensino podem ser adotados para aprimorar e enriquecer a experiência de aprendizado dos alunos (Mohammad, 2015).

Diante disso, diversas instituições estão buscando a aprendizagem baseada em problemas como uma solução para incentivar a criatividade e o pensamento crítico dos alunos (Damodharan \& Rengarajan, 2007; Silva, Cruz, \& Sahb, 2018), uma vez que o PBL está baseado no estudo de problemas propostos com o intuito de fazer com que o aluno estude determinados conteúdos e tem predominado como forma de possibilitar o aprendizado de conteúdos cognitivos e a integração de disciplinas (Silva et al., 2018). Além da aprendizagem baseada em problemas, existem vários exemplos de metodologias ativas que podem ser utilizadas em sala de aula, como aula expositiva dialogada, visita técnica, grupo de verbalização/grupo de observação, debate, seminário, filmes, storytelling, prática de campo, gamificação, mapas conceituais, mapas mentais, entre outras (Leal, Miranda, \& Casa Nova, 2017).

Barbosa (2018) também ressalta a possibilidade de se mesclar duas ou mais estratégias, uma vez que elas não precisam ser aplicadas isoladamente. Desta forma, é possível a utilização de aulas expositivas aliadas a debates, por exemplo, tendo o professor como mediador (Barbosa, 2018). Tendo em vista essa diversidade de metodologias que podem ser utilizadas, tanto isoladamente quanto em conjunto com outras, segue abaixo o Quadro 2, o qual apresenta uma síntese das metodologias inovadoras apresentadas.

\begin{tabular}{|l|l|}
\hline \multicolumn{1}{|c|}{ Metodologia } & \multicolumn{1}{c|}{ Síntese } \\
\hline Aula expositiva dialogada & Utiliza a pergunta como ferramenta para a construção do conhecimento. \\
\hline Debate & $\begin{array}{l}\text { Proporciona a discussão formal sobre um tema polêmico, contrapondo opiniões a fim de } \\
\text { esclarecer e detalhar o assunto em questão. }\end{array}$ \\
\hline Caso de ensino & $\begin{array}{l}\text { Permite a proposição de soluções para os problemas apresentados por meio da análise } \\
\text { de casos práticos. }\end{array}$ \\
\hline Gamificação & $\begin{array}{l}\text { Fornece feedback imediato sobre as decisões tomadas pelos jogadores, simulando o } \\
\text { acesso a problemas da vida real. }\end{array}$ \\
\hline $\begin{array}{l}\text { Grupo de verbalização/grupo de } \\
\text { observação }\end{array}$ & $\begin{array}{l}\text { Possibilita aos participantes um papel proativo na trajetória do conhecimento por meio } \\
\text { de dinâmicas em grupos. }\end{array}$ \\
\hline Mapa mental e mapa conceitual & $\begin{array}{l}\text { Utilizam palavras-chave para reativar as lembranças e desenvolver a memória e/ou } \\
\text { permitem uma visão do geral e do específico com relação aos conteúdos ministrados, } \\
\text { facilitando a construção de conhecimentos. }\end{array}$ \\
\hline
\end{tabular}




\begin{tabular}{|l|l|}
\hline $\begin{array}{l}\text { PBL (aprendizagem baseada em } \\
\text { problemas) }\end{array}$ & $\begin{array}{l}\text { Possibilita o aprendizado de conteúdos cognitivos e a integração de disciplinas } \\
\text { baseando-se no estudo de problemas. }\end{array}$ \\
\hline Prática de campo & Permite que o discente vivencie e aprenda por meio da prática. \\
\hline Sala de aula invertida & $\begin{array}{l}\text { Combina várias ferramentas visando a aprendizagem, permitindo, assim, a troca de } \\
\text { experiências entre os alunos durante a fase de autoaprendizagem. }\end{array}$ \\
\hline Seminário & Promove a discussão e o debate de temas, sob a direção do professor. \\
\hline Storytelling & Relaciona histórias ou músicas ao conteúdo estudado. \\
\hline Visita técnica & $\begin{array}{l}\text { Possibilita a visualização dos conceitos aprendidos e discutidos em sala de aula, } \\
\text { complementando o processo de ensino e aprendizagem. }\end{array}$ \\
\hline
\end{tabular}

Quadro 2. Síntese Metodologias de Ensino Inovadoras

Fonte: Adaptado de (Damodharan \& Rengarajan, 2007; Sorebo \& Haehre, 2012; Mohammad, 2015; Silva, 2015; Leal et al., 2017; Barbosa, 2018; Dai, Li, Liu, \& Lu, 2018; Nóbrega, David, \& Silva, 2018; Silva et al., 2018)

\subsection{Intenção}

A rápida evolução do segmento de educação superior no Brasil, tanto em quantidades de instituições quanto em novos cursos oferecidos, fez das escolhas educacionais dos indivíduos um importante objeto de estudo da sociologia e da educação (Martelli \& Santos, 2013). Nesse contexto, a escolha de um curso de graduação, enquanto processo decisório, ocorre de forma mais confiável à medida que o indivíduo possui maior e melhor informação sobre os cursos e profissões e sobre si próprio (Bomtempo, Silva, \& Freire, 2012). Além disso, com a contribuição da psicologia educacional, têm sido desenvolvidas pesquisas relacionadas à aprendizagem dos universitários visando compreender os fatores que influenciam as escolhas, expectativas e envolvimento dos estudantes com a sua formação (Farias, Francisco Jr., \& Ferreira, 2010).

Existe um claro interesse das IES na compreensão dos fatores que influenciam a escolha dos cursos e da instituição por parte dos alunos (Souza \& Reinert, 2009). Assim, a ideia é aumentar a convergência entre os motivos dos estudantes e os objetivos da instituição, além de conhecer as variáveis que podem gerar insatisfação por parte do corpo discente e prevenir possíveis movimentos evasivos decorrentes do não atendimento das expectativas dos estudantes (Souza \& Reinert, 2009). As IES devem identificar como os seus usuários ajustam suas crenças e atitudes sobre os atributos ofertados e como essas crenças e atitudes interferem na intenção comportamental dos futuros acadêmicos no processo de escolha do serviço (Sousa et al., 2013).

Desta forma, a intenção pertence ao domínio prático e está relacionada a uma vontade, um desejo, uma aspiração (Schio, 2009). Além disso, a intenção é o grau em que as pessoas formulam planos conscientes a fim de realizar ou não algum comportamento futuro determinado (Ramayah, Lee, \& Mohamad, 2010; Ramírez, Mariano, \& Salazar, 2014). Nesse contexto, as atitudes das pessoas demonstram sua motivação, pois interferem na decisão de querer ou não fazer alguma coisa de acordo com determinada situação (Trigwell, Ashwin, \& Millan, 2013).

\section{Procedimentos metodológicos}

O presente estudo buscou identificar a influência das metodologias de ensino adotadas sobre a intenção dos acadêmicos de uma Instituição de Ensino Superior da região Sul do Brasil em cursar um curso superior de tecnologia na área de gestão, tendo sido a amostra escolhida por conveniência, uma vez que foi utilizado um curso específico da área de gestão da Instituição. A pesquisa, assim, apresenta um enfoque quantitativo, caracterizando-se também como descritiva e aplicada. A pesquisa com enfoque quantitativo utiliza a coleta de dados visando estabelecer padrões e comprovar teorias (Sampiere, Collado, \& Lucio, 2013). Além disso, pela utilização de técnicas padronizadas de coleta de dados, a pesquisa descritiva objetiva especificar as 
características de uma determinada população ou fenômeno (Sampiere et al., 2013; Cooper \& Schindler, 2016). A pesquisa aplicada, por sua vez, objetiva utilizar os conhecimentos obtidos para a solução de problemas concretos do cotidiano (Cooper \& Schindler, 2016), uma vez que os resultados obtidos podem contribuir para a adoção de metodologias de ensino mais adequadas aos cursos de educação tecnológica.

Na pesquisa experimental deve haver a seleção das variáveis, a especificação dos níveis de tratamento e a definição das formas de controle e de observação dos efeitos para que os testes e análises dos dados possam ser realizados adequadamente (Cooper \& Schindler, 2016). Assim, o presente estudo foi desenvolvido por meio de um experimento baseado em cenários com a utilização de vinhetas. Para a realização deste experimento foi utilizado um cenário contendo uma variável dependente (intenção de cursar), e uma variável independente apresentada de duas formas (com metodologias tradicionais e com metodologias inovadoras). A realização do experimento deu-se de forma presencial no início do mês de abril de 2019. Os alunos do curso analisado foram convidados a participar do estudo durante as aulas de três disciplinas do semestre em que se encontravam. Nas ocasiões de realização das coletas, foram encontrados em sala de aula 85 alunos do curso, de um total de 152 alunos matriculados.

Foram utilizadas vinhetas no formato de folders para simulação das metodologias de ensino adotadas. As vinhetas continham as características do curso (apresentação, carga-horária e metodologias utilizadas no curso). Uma das versões, entregue ao primeiro grupo de acadêmicos, seguiu uma construção mais tradicional, seguindo um padrão de divulgação de folders de cursos de graduação. A versão entregue ao segundo grupo de acadêmicos, porém, apresentava uma proposta com apelo mais inovador, desde a apresentação do curso até as metodologias de ensino e ambientes de aprendizagem utilizados. As vinhetas foram atribuídas aleatoriamente aos participantes, garantindo que cada participante tivesse a mesma chance de receber cada uma das versões das vinhetas. Assim, foi possível analisar, por meio da manipulação da variável independente, os efeitos na variável dependente (Rungtusanatham, Wallin, \& Eckerd, 2011; Sampiere et al., 2013).

Logo após a apresentação das vinhetas, os acadêmicos responderam ao questionário sobre a intenção de realização do curso. O questionário apresentava em sua parte introdutória nove itens relacionados a informações demográficas (como idade, sexo, estado civil, renda, experiência profissional na área de gestão e semestre atual do curso) e mais duas variáveis que visavam captar a percepção do respondente quanto à proposta do curso apresentado na vinheta. Na parte central, o instrumento possuía 20 variáveis, as quais foram adaptadas de Bomtempo, Silva e Freire (2012), Piteira, Costa e Aparicio (2017), e Souza (2009) e mensuradas por meio de uma escala do tipo Likert de 5 pontos (variando de $1=$ discordo totalmente a $5=$ concordo totalmente). Além disso, apresentava no final mais duas questões, uma relacionada à disposição de realizar o curso e outra relacionada ao cenário apresentado na vinheta.

Foram observadas cinco variáveis com relação aos dois grupos (P1, P2, I, D e R), onde P1 e P2 estão relacionadas à proposta de curso apresentada (P1 indica se a proposta despertou o interesse do aluno e P2, se o aluno recomendaria o curso), I é a intenção de cursar (medida pela variável média por meio de 20 itens), D é a disposição em cursar o curso e R é a avaliação do realismo do cenário apresentado. As características dos respondentes foram analisadas de forma combinada a fim de verificar a existência de diferenças entre os dois grupos analisados. Para verificar a confiabilidade dos construtos, foi utilizada a medida de consistência interna alfa de Cronbach, a qual, segundo Hernandez, Basso, \& Brandão, (2014), é bastante utilizada em estudos experimentais.

Os dados da pesquisa foram analisados de forma quantitativa, sendo utilizada a técnica estatística de 
análise de variância (ANOVA), que é utilizada quando se pretende identificar se as médias entre dois ou mais grupos diferem entre si (Agresti \& Finlay, 2012; Hair Jr., Babin, Money, \& Samouel, 2005). O teste ANOVA compara duas estimativas independentes da variância para a variável dependente, onde uma representa a variabilidade geral dos respondentes dentro dos grupos e a outra indica as diferenças entre os grupos (Hair Jr., Black, Babin, Anderson, \& Tatham, 2009). Após a coleta, os dados foram tabulados e depois analisados por meio do software SPSS (Statistical Package for the Social Sciences).

\section{Análise dos dados}

A amostra foi composta por 85 acadêmicos de um curso superior de tecnologia da área de gestão de uma IES da região sul do Brasil e foi escolhida por conveniência, tendo em vista a preferência por um curso em específico. Conforme informações da Instituição, o curso contava com 152 alunos matriculados no primeiro semestre de 2019 nas três disciplinas cujos alunos fizeram parte do estudo. As vinhetas foram atribuídas de forma aleatória entre os respondentes, sendo que após a distribuição, contando as três turmas que participaram da pesquisa, 40 acadêmicos receberam a vinheta com metodologia tradicional e 45 receberam a vinheta com metodologia inovadora.

Com relação à faixa etária, a maior parte dos respondentes $(54,1 \%)$ possui entre 18 e 24 anos; 43,5\% dos respondentes possuem entre 25 e 50 anos e 2,4\% possuem mais de 50 anos. Houve a predominância de respondentes do sexo masculino (58,8\%), contra 40,0\% de respondentes do sexo feminino. Além disso, 1 respondente (1,2\%) se absteve desta resposta. Quanto ao estado civil, 62,4\% dos acadêmicos responderam que são solteiros; $34,1 \%$ são casados ou possuem relacionamento fixo e 3,5\% são divorciados ou outros.

De acordo com os dados apresentados, a maior parte dos respondentes está cursando o terceiro semestre $(37,7 \%) ; 31,8 \%$ dos respondentes estão cursando o primeiro semestre; $3,5 \%$ o quarto semestre; e $27 \%$ estão cursando o quinto semestre. Com relação à experiência profissional na área de gestão, a maioria dos respondentes $(61,2 \%)$ não possui experiência na área, contra 38,8\% que possuem experiência. Dos respondentes que possuem experiência na área de gestão, a maior parte possui até 1 ano de experiência $(10,6 \%)$ ou de 2 a 4 anos (11,7\%). Entre os que possuem de 5 a 10 anos de experiência foram contabilizados 9,4\%, enquanto 7,1\% possuem mais de 10 anos de experiência na área de gestão.

Com relação à renda, a grande maioria está na faixa de até $\mathrm{R} \$ 2.499,99(43,5 \%)$ ou de $\mathrm{R} \$ 2.500,00 \mathrm{a}$ $\mathrm{R} \$ 4.999,99$ (40,0\%); 13\% dos respondentes possuem renda entre $\mathrm{R} \$ 5.000,00$ e $\mathrm{R} \$$ 9.999,99; e 3,5\% possuem renda acima de R \$10.000,00 ou não responderam a este item. Quanto à composição das famílias, a distribuição foi bem uniforme entre os grupos de 2, 3 e 4 pessoas, com predominância para o grupo de 3 pessoas. Do total, $7,1 \%$ das famílias são formadas por apenas uma pessoa; $24,7 \%$ por 2 pessoas; $29,4 \%$ por 3 pessoas; 23,5\% por 4 pessoas e 15,3\% por 5 ou mais pessoas. Além disso, de acordo com os dados apresentados na tabela 4, a maior parte dos respondentes mora com os pais $(43,5 \%)$ ou com o cônjuge $(34,1 \%)$. Do restante, $14,1 \%$ moram sozinhos e $8,3 \%$ moram com amigos ou outros.

Quanto a consistência dos dados o alfa de Cronbach obtido foi 0,847 , indicando que os construtos são consistentes com as mensurações (Hair et al., 2009). Ao longo da análise dos dados foi possível observar quanto à intenção de cursar, conforme dados disponíveis na tabela 1, que a média do método inovador foi 3,7078 , sendo superior à média apresentada no método tradicional, que foi 3,4425. O grupo que recebeu a vinheta com metodologia inovadora teve maior média quando analisadas as variáveis relativas à proposta apresentada (P1 e P2), o que pode indicar que os acadêmicos possuem maior probabilidade de se interessar e/ ou indicar para alguém um curso com este tipo de metodologia. De encontro a este achado, porém, tem-se que 
o grupo com metodologia tradicional tem maior disposição para cursar o curso apresentado do que o grupo inovador, o que pode indicar que, apesar do interesse pelo curso inovador, os acadêmicos ainda não estão acostumados com a oferta de cursos com este tipo de metodologia.

Apesar da diferença entre as médias, no entanto, a análise de variância não indicou diferença significativa entre os dois grupos com relação às duas questões relativas à proposta de curso apresentada: interesse pelo curso dada a metodologia apresentada (P1) e recomendação do curso para um colega, amigo ou familiar (P2); bem como com relação às questões referentes à disposição de realizar o curso (D) e ao realismo do cenário apresentado (R). Neste último aspecto, as duas propostas não apresentaram diferenças significantes no realismo, validando a manipulação.

Quando analisada a intenção de cursar o curso apresentado, porém, foi encontrado como resultado que as médias diferem de forma significativa entre os dois grupos (com valor de 0,029), conforme pode ser observado na tabela 1 .

Tabela 1

ANOVA Vinhetas

\begin{tabular}{|c|c|c|c|c|c|c|}
\hline & Vinhetas & $\begin{array}{l}\text { Soma dos } \\
\text { quadrados }\end{array}$ & $\begin{array}{l}\text { Graus } \\
\text { liberd. }\end{array}$ & $\begin{array}{l}\text { Média dos } \\
\text { quadrados }\end{array}$ & $\begin{array}{c}\text { Estatística } \\
\text { F }\end{array}$ & $\begin{array}{c}\text { Valor } \\
\mathbf{P}\end{array}$ \\
\hline \multirow{3}{*}{$\begin{array}{l}\text { Proposta } 1 * \\
\text { vinheta }\end{array}$} & Entre os Grupos (Combinados) & 1,237 & 1 & 1,237 & 1,085 &, 301 \\
\hline & Dentro dos Grupos & 94,575 & 83 & 1,139 & & \\
\hline & Total & 95,812 & 84 & & & \\
\hline \multirow{3}{*}{$\begin{array}{l}\text { Proposta } 2 * \\
\text { vinheta }\end{array}$} & Entre os Grupos (Combinados) & 1,324 & 1 & 1,324 & 1,425 & ,236 \\
\hline & Dentro dos Grupos & 77,100 & 83 & ,929 & & \\
\hline & Total & 78,424 & 84 & & & \\
\hline \multirow{3}{*}{$\begin{array}{l}\text { Disposição * } \\
\text { vinheta }\end{array}$} & Entre os Grupos (Combinados) &, 137 & 1 &, 137 &, 034 &, 854 \\
\hline & Dentro dos Grupos & 334,686 & 83 & 4,032 & & \\
\hline & Total & 334,824 & 84 & & & \\
\hline \multirow{3}{*}{$\begin{array}{l}\text { Realismo * } \\
\text { vinheta }\end{array}$} & Entre os Grupos (Combinados) &, 871 & 1 &, 871 & ,899 & ,346 \\
\hline & Dentro dos Grupos & 80,353 & 83 & ,968 & & \\
\hline & Total & 81,224 & 84 & & & \\
\hline \multirow{3}{*}{$\begin{array}{l}\text { Intenção de } \\
\text { cursar * vinheta }\end{array}$} & Entre os Grupos (Combinados) & 1,490 & 1 & 1,490 & 4,919 & 029 \\
\hline & Dentro dos Grupos & 25,148 & 83 & ,303 & & \\
\hline & Total & 26,638 & 84 & & & \\
\hline
\end{tabular}

Os resultados também indicam que a intenção de cursar para o grupo inovador é superior à do grupo tradicional, conforme gráfico 1, o que pode indicar que os acadêmicos de nível superior estão tendendo a preferir formas de aprendizagem mais inovadoras. 


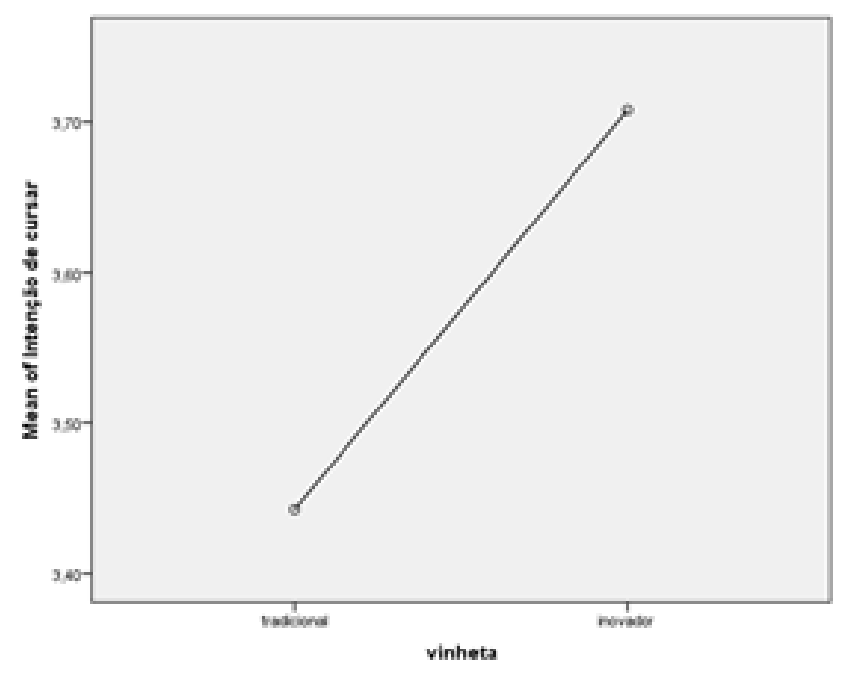

Gráfico 1. Relação entre os grupos e a intenção de cursar

Esse resultado é corroborado por pesquisas anteriores, onde a utilização de uma metodologia de ensino mais ativa é um fator que facilita o aprendizado (Morozini et al., 2007). Além disso, a implementação de metodologias mais ativas aumenta a participação e envolvimento dos alunos (Vigaray et al., 2010). As metodologias mais ativas, como a sala de aula invertida, por exemplo, despertam um maior interesse e participação dos alunos (Nóbrega et al., 2018). A adoção de metodologias ativas, com a adoção de novas e variadas ferramentas e procedimentos durante as aulas, são mais efetivas para a aprendizagem na percepção dos alunos (Brito \& Campos, 2019).

Com relação aos fatores demográficos analisados, os fatores faixa etária, sexo, estado civil, experiência profissional, renda, número de pessoas na família e com quem mora, não apresentaram diferença significativa, e por esse motivo não serão discutidos nesta seção. Já para os fatores semestre do curso e tempo de experiência profissional na área de gestão foram encontradas diferenças significativas entre os grupos, as quais serão apresentadas a seguir.

Com relação ao semestre que os respondentes estavam cursando, conforme a tabela 2, os resultados indicam que as médias de P1 e disposição tendem a ser maiores quanto maior o semestre. As médias de P2, por outro lado, tendem a ser menores quanto maior o semestre. Com relação à intenção de realização do curso apresentado, observa-se que a média dos respondentes do $3^{\circ}$ semestre é maior que a dos demais semestres, principalmente o $4^{\mathrm{o}}$ semestre, que possui a menor média. O $4^{\mathrm{o}}$ semestre, inclusive, destoou dos demais semestres também quanto à percepção de realismo do cenário apresentado, uma vez que a média foi aumentando até o referido semestre e depois diminuiu. Com relação à significância entre as diferenças encontradas, porém, os dados da tabela 2 indicam que as médias dos dois grupos, apesar de diferentes, não apresentaram diferença significativa para as variáveis P1, P2, R e I. Por outro lado, com relação à disposição de cursar o curso apresentado, foram observadas diferenças significativas entre as médias dos dois grupos, com significância de 0,023 .

\section{Tabela 2}

ANOVA Semestre do Curso 


\begin{tabular}{|c|c|c|c|c|c|c|}
\hline \multirow{3}{*}{$\begin{array}{l}\text { Proposta } 1 * \\
\text { semestre_curso }\end{array}$} & Entre os Grupos (Combinados) & 1,600 & 3 &, 533 & ,459 & ,712 \\
\hline & Dentro dos Grupos & 94,212 & 81 & 1,163 & & \\
\hline & Total & 95,812 & 84 & & & \\
\hline \multirow{3}{*}{$\begin{array}{l}\text { Proposta } 2 * \\
\text { semestre_curso }\end{array}$} & Entre os Grupos (Combinados) & 1,648 & 3 &, 549 &, 580 & ,630 \\
\hline & Dentro dos Grupos & 76,775 & 81 & ,948 & & \\
\hline & Total & 78,424 & 84 & & & \\
\hline \multirow{3}{*}{$\begin{array}{l}\text { Disposição * } \\
\text { semestre_curso }\end{array}$} & Entre os Grupos (Combinados) & 36,997 & 3 & 12,332 & 3,354 & 023 \\
\hline & Dentro dos Grupos & 297,827 & 81 & 3,677 & & \\
\hline & Total & 334,824 & 84 & & & \\
\hline \multirow{3}{*}{$\begin{array}{l}\text { Realismo * } \\
\text { semestre_curso }\end{array}$} & Entre os Grupos (Combinados) & 3,839 & 3 & 1,280 & 1,340 & 267 \\
\hline & Dentro dos Grupos & 77,384 & 81 & ,955 & & \\
\hline & Total & 81,224 & 84 & & & \\
\hline \multirow{3}{*}{$\begin{array}{l}\text { Intenção de } \\
\text { cursar* } \\
\text { semestre_curso }\end{array}$} & Entre os Grupos (Combinados) & 1,147 & 3 & ,382 & 1,215 & ,310 \\
\hline & Dentro dos Grupos & 25,491 & 81 & ,315 & & \\
\hline & Total & 26,638 & 84 & & & \\
\hline
\end{tabular}

Abaixo, o gráfico 2 apresenta a relação entre o semestre atual que os respondentes estão cursando e a disposição de cursar o novo curso, indicando que, quanto maior o semestre, maior é a disposição dos respondentes de cursar o curso proposto.

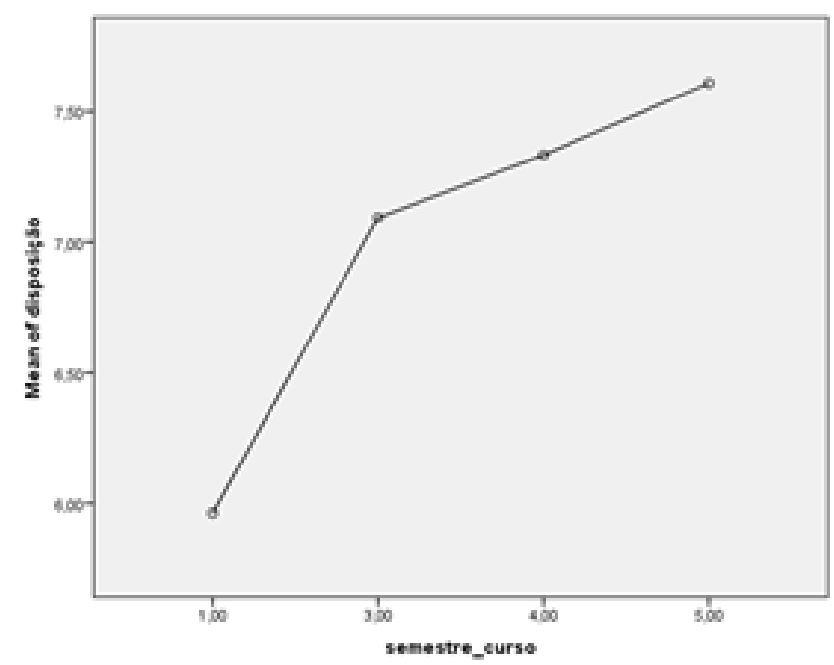

Gráfico 2. Relação entre o semestre do curso e a disposição

Com relação ao tempo de experiência, conforme dados disponíveis na tabela 3, as médias tendem a ser maiores para P1, P2, I, D e R para quem possui mais de 4 anos de experiência profissional na área de gestão. Por outro lado, quem possui de 2 a 4 anos de experiência possui a menor média em todas as variáveis analisadas. Para quem não possui experiência, as médias tendem a ser maiores em relação a quem possui até um ano de experiência para P1, P2 e R, e menores para disposição e intenção. Conforme os dados disponíveis na tabela 3, com relação ao tempo de experiência, os grupos não apresentaram diferença significativa para as variáveis P1, P2 e I. Porém, foram observadas diferenças significativas entre as médias dos dois grupos com relação à disposição de cursar o curso apresentado $(0,001)$ e ao realismo do cenário $(0,008)$. 


\begin{tabular}{|c|c|c|c|c|c|c|}
\hline \multicolumn{2}{|c|}{ Tempo de Experiência } & \multirow{2}{*}{$\begin{array}{c}\begin{array}{c}\text { Soma dos } \\
\text { quadrados }\end{array} \\
9,496\end{array}$} & \multirow{2}{*}{$\begin{array}{c}\begin{array}{c}\text { Graus } \\
\text { liberd. }\end{array} \\
5\end{array}$} & \multirow{2}{*}{$\begin{array}{c}\begin{array}{c}\text { Média dos } \\
\text { quadrados }\end{array} \\
1,899\end{array}$} & \multirow{2}{*}{$\begin{array}{c}\begin{array}{c}\text { Estatística } \\
\mathbf{F}\end{array} \\
1,738\end{array}$} & \multirow{2}{*}{$\begin{array}{c}\begin{array}{c}\text { Valor } \\
\mathbf{P}\end{array} \\
, 136\end{array}$} \\
\hline Proposta $1 *$ & Entre os Grupos (Combinados) & & & & & \\
\hline tempo_experiência & Dentro dos Grupos & 86,316 & 79 & 1,093 & & \\
\hline & Total & 95,812 & 84 & & & \\
\hline \multirow{3}{*}{$\begin{array}{l}\text { Proposta } 2 * \\
\text { tempo_experiência }\end{array}$} & Entre os Grupos (Combinados) & 6,966 & 5 & 1,393 & 1,540 &, 187 \\
\hline & Dentro dos Grupos & 71,458 & 79 & ,905 & & \\
\hline & Total & 78,424 & 84 & & & \\
\hline \multirow{3}{*}{$\begin{array}{l}\text { Disposição * } \\
\text { tempo_experiência }\end{array}$} & Entre os Grupos (Combinados) & 72,698 & 5 & 14,540 & 4,382 &, 001 \\
\hline & Dentro dos Grupos & 262,126 & 79 & 3,318 & & \\
\hline & Total & 334,824 & 84 & & & \\
\hline \multirow{3}{*}{$\begin{array}{l}\text { Realismo * } \\
\text { tempo_experiência }\end{array}$} & Entre os Grupos (Combinados) & 14,333 & 5 & 2,867 & 3,385 &, 008 \\
\hline & Dentro dos Grupos & 66,891 & 79 &, 847 & & \\
\hline & Total & 81,224 & 84 & & & \\
\hline \multirow{3}{*}{$\begin{array}{l}\text { Intenção de cursar * } \\
\text { tempo_experiência }\end{array}$} & Entre os Grupos (Combinados) & 1,021 & 5 & ,204 &, 630 & 677 \\
\hline & Dentro dos Grupos & 25,617 & 79 &, 324 & & \\
\hline & Total & 26,638 & 84 & & & \\
\hline
\end{tabular}

Conforme pode ser observado no gráfico 3, quanto maior o tempo de experiência na área de gestão, maior é a disposição em cursar o curso apresentado na vinheta, o que, de acordo com Bomtempo et al., (2012), pode estar relacionado ao fato de a aproximação com a área administrativa se constituir em um forte fator de influência na escolha do curso. A exceção, porém, é o intervalo entre dois e quatro anos de experiência, no qual as médias tendem a diminuir quanto maior o tempo de experiência.

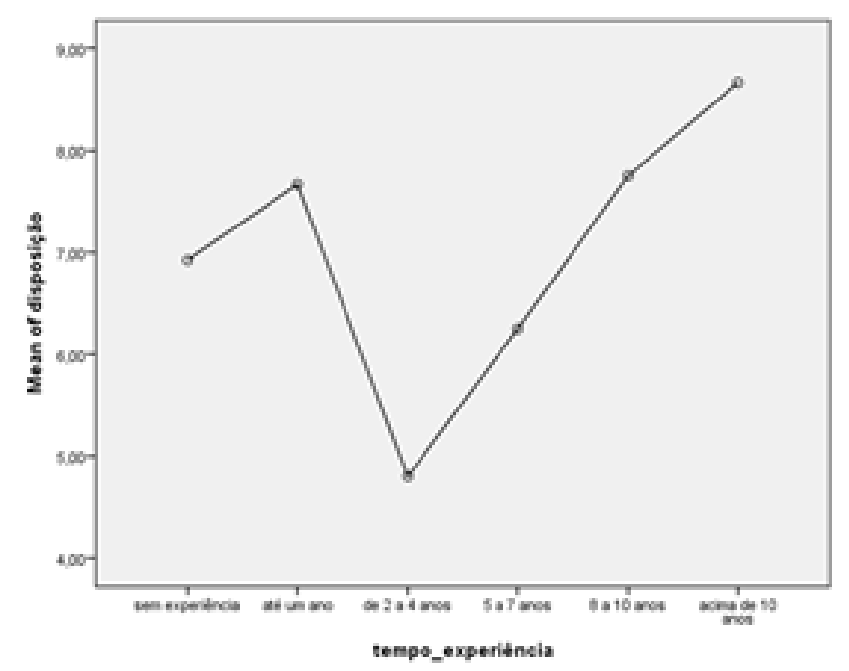

Gráfico 3. Relação entre o tempo de experiência e a disposição

No gráfico 4, os resultados demonstram que quanto maior o tempo de experiência na área de gestão, menor é a percepção sobre o realismo do cenário apresentado na vinheta (o quão próximo da realidade foi considerado o folder apresentado), com exceção dos grupos que possuem entre cinco e dez anos de experiência, nos quais a percepção sobre o realismo do cenário apresentado aumentou com um maior tempo de experiência. 


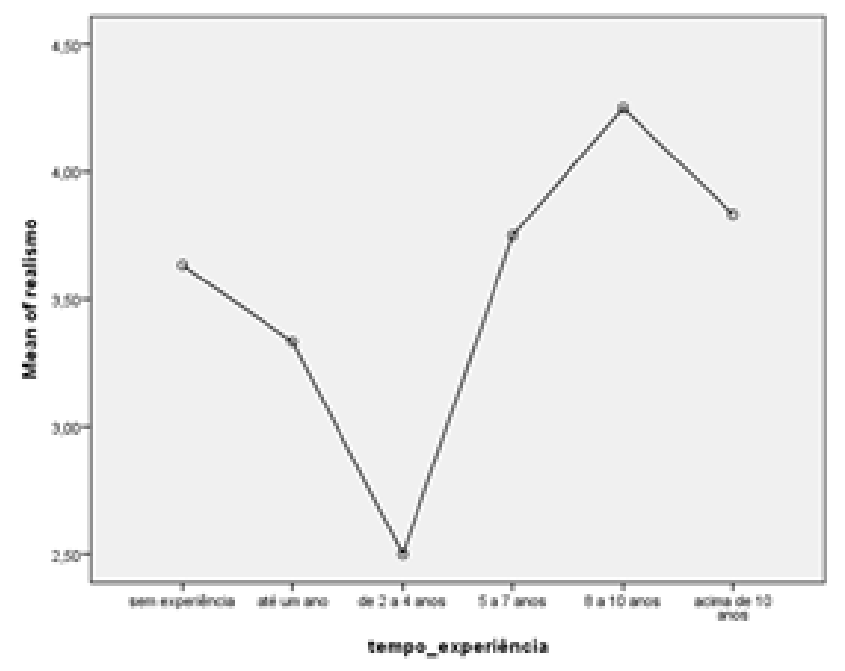

Gráfico 4. Relação entre o tempo de experiência e o realismo do cenário

Com relação ao objetivo da pesquisa, conforme pode ser observado pela análise dos dados contida nessa seção, em síntese, os resultados demonstram que a intenção de cursar o curso apresentado nas vinhetas apresentou diferença significativa entre os grupos, sendo maior para o grupo inovador. Além disso, quando levado em consideração o semestre que os respondentes estão cursando e o tempo de experiência na área de gestão, foram encontradas diferenças significativas entre os dois grupos com relação à disposição de cursar o curso e a percepção sobre o realismo do cenário apresentado.

\section{Considerações finais}

As mudanças ocorridas no ambiente acadêmico nas últimas décadas e o consequente aumento no número de alunos matriculados nas IES tem fortalecido a necessidade de estudos sobre as mudanças e melhorias que podem ser aplicadas no processo de ensino-aprendizagem visando um melhor aproveitamento por parte dos alunos. Nesse contexto, o presente estudo buscou identificar a influência das metodologias de ensino adotadas e a intenção dos acadêmicos em cursar um curso superior de tecnologia na área de gestão.

As análises realizadas demonstraram uma diferença significativa entre as médias dos dois grupos analisados com relação à intenção de cursar o curso apresentado. Os resultados também indicam que as médias foram maiores para o grupo que recebeu o folder com metodologia inovadora, o que vai ao encontro de estudos como o de Vigaray et al. (2010); Nóbrega et al. (2018); Brito e Campos (2019).

Com relação aos fatores demográficos analisados, apenas dois apresentaram significância para as variáveis analisadas: semestre que os respondentes estavam cursando em seu curso atual e tempo de experiência profissional na área de gestão, indicando que o avanço no curso e o tempo de atuação no mercado de trabalho na área de gestão, influenciam a percepção dos respondentes quanto à disposição de realizar um novo curso de tecnologia na área de gestão. Nesse contexto, diante dos resultados obtidos, foi elaborada uma proposta de intervenção para a IES participante do estudo, com a sugestão de utilização de metodologias ativas, em conjunto com as metodologias tradicionais já utilizadas, visando obter um melhor aproveitamento, interesse e aprendizado por parte dos alunos.

Embora estudos envolvendo este tema não sejam recentes, tem demonstrado um interesse crescente tanto no meio educacional, uma vez que as IES buscam cada vez mais compreender os fatores que influenciam a escolha dos alunos, quanto no meio empresarial, tendo em vista que a utilização de novos modelos de ensino 
tende a possibilitar a formação de um profissional melhor preparado para o mercado de trabalho. Assim, o estudo busca contribuir para a melhoria do serviço educacional de nível tecnológico prestado pelas IES, com o intuito de atender da melhor forma as expectativas de seu público alvo e oferecer um ensino de qualidade e cada vez mais voltado à realidade do mercado.

Além disso, uma importante contribuição do presente estudo é a confirmação de que os estudantes analisados, em sua maioria, estão tendendo a buscar caminhos mais desafiadores e estimulantes na construção de seu conhecimento, o que possibilita cada vez mais a utilização de modelos de ensino inovadores e que permitam um maior protagonismo do aluno, principalmente em um cenário tão dinâmico como o educacional.

Como limitações do estudo, tem-se que os resultados representam a realidade do curso analisado, não podendo ser generalizada para os demais cursos de tecnologia, da área de gestão ou não. Por isso, inclusive, sugere-se a realização de novos estudos visando identificar a intenção dos acadêmicos com relação às metodologias de ensino adotadas em outros cursos superiores de tecnologia não só na área de gestão, como também em outras áreas, uma vez que estes cursos têm aplicação eminentemente prática e metodologias mais ativas tendem a desenvolver um melhor preparo dos acadêmicos para o mercado de trabalho. Também se sugere a realização de experimentos não só de campo, mas também de laboratório, com a análise da utilização de metodologias inovadoras em cursos superiores de tecnologia, visando identificar se, na prática, o comportamento dos acadêmicos é o mesmo identificado quando da experimentação com cenários, como foi o caso deste estudo.

\section{Referências}

Agresti, A. \& Finlay, B. (2012). Métodos estatísticos para as ciências sociais (4a ed.). Porto Alegre: Penso.

Almeida, L. S., Araújo, A. M., \& Martins, C. (2016). Transição e adaptação dos alunos do $1^{\circ}$ ano: variáveis intervenientes e medidas de atuação. Almeida, L. S., \& Castro, R.V. (Orgs.). Ser estudante no ensino superior: o caso dos estudantes do $1^{\mathrm{o}}$ ano. Braga: Universidade do Minho.

Almeida, L. S., Araújo, A. M., \& Martins, C. (2016). Transição e adaptação dos alunos do $1^{\text {o }}$ ano: Variáveis intervenientes e medidas de atuação. Seminário "Ser Estudante no Ensino Superior: O caso dos estudantes do 1. ${ }^{\circ}$ ano", 146-164.

Alves, E. B., Rolon, V. E. K., Petry, A., Farias, A. P., Dick, L. C., \& Melo, T. Y. M. (2018). Metodologias ativas no foco da discussão da educação em administração: um trabalho bibliométrico de estudos de 2009 a 2017. Encontro de Iniciação Científica, Fórum Científico e Seminário do Programa Institucional de Bolsa de Iniciação a Docência, Curitiba, PR, Brasil, 14.

Andere, M. A. (2007). Aspectos da formação do professor de ensino superior de Ciências Contábeis: Uma análise dos programas de pós-graduação. Dissertação de Mestrado, Universidade de São Paulo, São Paulo, SP, Brasil.

Araújo, A. M., Santos, A. A., Noronha, A. P., Zanon, C., Ferreira, J. A., Casanova, J. R., \& Almeida, L. S. 
(2016). Dificuldades antecipadas de adaptação ao ensino superior: um estudo com alunos do primeiro ano. Revista de estudios e investigación en psicología y educación, 3(2), 102-111.

Barbosa, C. C. (2018). A contribuição das metodologias ativas como recurso didático no ensino superior. Prometeu- Projeto de meios tecnológicos em educação universitária, 4(4), 36-41.

Biggs, J. B. (1999). Teaching for quality learning at university: what the student does. Buckingham: Society for Research into Higher Education.

Bomtempo, M. S., Silva, D., \& Freire, O. B. L. (2012). Motivos da escolha do curso de administração de empresas por meio da modelagem de equações estruturais. Pretexto, 13(3), 108-129.

Brandão, J. M. F., Cavalcante, E. D. C., \& Temoteo, J. A. G. (2014). O processo de aprendizagem de alunos de turismo e hotelaria sob a perspectiva andragógica. Revista Brasileira de Pesquisa em Turismo, 8(3), 531-551.

Brito, C. A. F., \& Campos, M. Z. (2019). Facilitando o processo de aprendizagem no ensino superior: o papel das metodologias ativas. Revista Ibero-Americana de Estudos em Educação, 14(2), 371-387.

Cooper, D. R., \& Schindler, P.S. (2016). Métodos de pesquisa em administração (12a ed.). Porto Alegre: AMGH.

Dai, Y., Li, T., Liu, A., \& Lu, S. (2018). P2P Platform for Peer Instruction in Flipped Classroom. In 2018 ASEE Annual Conference \& Exposition

Damodharan, V. S., \& Rengarajan, V. (2007). Innovative methods of teaching. In Learning Technologies and Mathematics Middle East Conference, Sultan Qaboos University, Muscat, Oman (pp. 1-16).

Farias, S. A., Francisco Jr., W. E., \& Ferreira, L. H. (2010). Motivação na escolha de um curso universitário: a valorização do diploma de nível superior nos cursos de Licenciatura em Química. Encontro Nacional de Ensino de Química-ENEQ, Brasília, DF, Brasil, 15.

Favretto, J., \& Moretto, C. F. (2013). Os cursos superiores de tecnologia no contexto de expansão da educação superior no Brasil: a retomada da ênfase na educação profissional. Educação \& Sociedade, 34(123), 407-424.

Ferraz, A. P. C. M., \& Belhot, R. V. (2010). Taxonomia de Bloom: revisão teórica e apresentação das adequações do instrumento para definição de objetivos instrucionais. Gest. Prod. 17 (2), 421-431.

Hair Jr., J. F., Babin, B., Money, A. H., \& Samouel, P. (2005). Fundamentos de métodos de pesquisa em administração. Porto Alegre: Bookman.

Hair Jr., J. F., Black, W. C., Babin, B. J., Anderson, R. E., \& Tatham, R. L. (2009). Análise multivariada de dados. Porto Alegre: Bookman. 
Hernandez, J. M. C., Basso, K., \& Brandão, M. M. (2014). Pesquisa experimental em marketing. Revista Brasileira de Marketing - ReMark, 13(2), 98-117.

Krüger, L. M., \& Ensslin, S. R. (2013). Método tradicional e método construtivista de ensino no processo de aprendizagem: uma investigação com os acadêmicos da disciplina Contabilidade III do curso de Ciências Contábeis da Universidade Federal de Santa Catarina. Organizações em Contexto, 9(18), 219-270.

Kuri, N. P., Silva, A. N. R., \& Pereira, M. A. (2006). Estilos de aprendizagem e recursos da hipermídia aplicados no ensino de planejamento de transportes. Revista Portuguesa de Educação, 19(2), 111-137.

Leal, E. A., Miranda, G. J., \& Casa Nova, S. P. C. (2017). Revolucionando a sala de aula: Como envolver o estudante aplicando as técnicas de metodologias ativas de aprendizagem. (1a ed.). São Paulo: Atlas.

Marques, A. P. A. Z., Message, C. P., Gitahy, R. R. C., \& Souza, S. O. (2018). A experiência da aplicação da metodologia ativa team based learning aliada a tecnologia no processo de ensino e de aprendizagem. Congresso Internacional de Educação e Tecnologias - Encontro de Pesquisadores em Educação a Distância CIET ENPED.

Martelli, P. A. A., \& Santos, A. R. J. (2013). Fatores que influenciam o processo de escolha do currículo na educação superior. Jornada de Didática e Seminário de Pesquisa do CEMAD, Londrina, PR, Brasil, 2.

Mello, S. P. T. (2007). Competências requeridas - competências adquiridas: o curso superior de Tecnologia em Sistemas de Telecomunicações do Centro Federal de Educação Tecnológica Pelotas - RS no contexto das mudanças advindas da reforma da educação profissional. Tese de Doutorado, Universidade Federal do Rio Grande do Sul, Porto Alegre, RS, Brasil.

Ministério da Educação (MEC). Secretaria de Educação Profissional e Tecnológica. (2016). Catálogo Nacional dos Cursos Superiores de Tecnologia. Brasília: MEC.

Mohammad, A. (2015). Experimental Methods of teaching business studies:

practical approaches beyond lecturing. International Journal of Core Engineering \& Management, 1(12), 5973.

Morozini, J. F., Cambruzzi, D., \& Longo, L. (2007). Fatores que influenciam o fator ensino aprendizagem no curso de ciências contábeis do ponto de vista acadêmico. Revista Capital Científico-Eletrônica, 5(1), 87-102.

Nóbrega, P. P., David, P. B., \& Silva, A. S. R. (2018). Sala de aula invertida e fatores intervenientes da aprendizagem: experiência em uma instituição federal de ensino superior com uma turma de alunos de graduação. Revista Paidéi@, 10(18), s.n.

Piteira, M., Costa, C.J., \& Aparicio, M. (2017). Canoe e Fluxo: Determinantes na adoção de curso de programação online gamificado. RISTI - Revista Ibérica de Sistemas e Tecnologias de Informação,25, 34-53. 
Ramayah, T., Lee, J. W. C., \& Mohamad, O. (2010). Green product purchase intention: Some insights from a developing country. Resources, Conservation and Recycling, 54 (12), 1419-1427.

Ramírez, P. E., Mariano, A. M., \& Salazar, E. A. (2014). Propuesta metodológica para aplicar modelos de ecuaciones estructurales con PLS: El caso del uso de las bases de datos científicas en estudiantes universitarios. Revista ADMpg Gestão Estratégica, 7(2), s.n.

Ramos, A., Delgado, F., Afonso, P., Cruchinho, A., Pereira, P., Sapeta, P., \& Ramos, G. (2013). Implementação de novas práticas pedagógicas no ensino superior. Revista Portuguesa de Educação, 26(1), 115-141.

Rungtusanatham, M., Wallin, C., \& Eckerd, S. (2011). The vignette in a scenario-based role-playing experiment. Journal of Supply Chain Management, 47(3), 9-16.

Sampieri, R. H., Collado, C. F., \& Lucio, M. P. B. (2013). Metodologia de pesquisa (5a ed.). Porto Alegre: Penso.

Schio, S. M. (2009). Aristóteles e ação humana. Conjectura, 14(1), 77-91.

Signori, G. G., Guimarães, J. C. F. D., Severo, E. A., \& Rotta, C. (2018). Gamification as an innovative method in the processes of learning in higher education institutions. International Journal of Innovation and Learning, 24(2), 115-137.

Silva, A. J. C., Cruz, S. R. M., \& Sahb, W. F. (2018). Metodologias ativas no Ensino Superior: uma proposta de oficina sobre aprendizagem por pares; sala de aula invertida; aprendizagem baseada em problema e rotação por estações de trabalho. Simpósio Tecnologias e Educação a Distância no Ensino Superior, Belo Horizonte, MG, Brasil, 1.

Silva, E. S. (2015). Mapas conceituais: propostas de aprendizagem e avaliação. Administração: ensino e pesquisa, $16(4), 785-815$.

Silva, G. da. (2014). O impacto da (des) motivação nos resultados estudantis. Monografia de Especialização, Universidade Federal do Paraná, Curitiba, PR, Brasil.

Silva, M. J. (2017). Abordagens tradicional e ativa: uma análise da prática partir da vivência no estágio supervisionado em docência. Seminário Internacional de Representações Sociais, Subjetividade e EducaçãoSIRSS, Curitiba, PR, Brasil, 4.

Sorebo, O., \& Haehre, R. (2012). Investigating students' perceived discipline relevance subsequent to playing educational computer games: a personal interest and self-determination theory approach. Scandinavian Journal of Educational Research, 56(4), 345-362. 
Sousa, A. M. R., Neto, A. R., \& Fontenele, R. E. S. (2013). Determinantes da intenção da escolha do ensino superior privado: uma perspectiva da teoria do comportamento planejado. Revista Eletrônica de Ciência Administrativa, 12(3), 367-378.

Souza, R. R. B. (2009). Intenção de escolha de ensino superior privado à luz da Teoria do Comportamento Planejado. Dissertação de Mestrado, Universidade Federal da Paraíba, João Pessoa, PB, Brasil.

Souza, S. A. de., \& Reinert, J. N. (2009). Motivação para entrada e permanência nos cursos de graduação em Administração da Universidade Federal de Mato Grosso do Sul. Anais Encontro da Anpad, São Paulo, SP, Brasil, 33.

Tabile, A. F., \& Jacometo, M. C. D. (2017). Fatores influenciadores no processo de aprendizagem: um estudo de caso. Revista Psicopedagogia, 34(103), 75-86.

Takahashi, A. R. W. (2010). Cursos superiores de tecnologia em gestão: reflexões e implicações da expansão de uma (nova) modalidade de ensino superior em administração no Brasil. Revista de Administração Pública, 44(2), 385-414.

Teixeira, M. C. (2015). Metodologia do ensino superior. Paraná: Unicentro.

Teles, A. A., Silva, J. N. G., \& Maciel, T. M. M. (2012). A inserção de novas metodologias no processo de ensino/aprendizagem em geografia. Anais VI Colóquio Internacional - Educação e Contemporaneidade, São Cristovão, RJ, Brasil.

Trigwell, K., Ashwin, P., \& Millan, E. S. (2013). Evoked prior learning experience and approach to learning as predictors of academic achievement. British Journal of Educational Psychology, 83(3), 363-378.

Vigaray, M. D. J., Lopez, J. J., Peris, J. E., Martínez, L. Y. C., Cuevas, J., Posadas, J. A., \& Vallés, M. L. (2010). Teaching methods: study and results in several modules of business studies. Proceedings of EDULEARN10 Conference, Barcelona, Spain.

${ }^{\mathrm{i}} \mathrm{O}$ instrumento de coleta e as vinhetas estão à disposição mediante solicitação aos autores. 